\title{
In Situ Lorentz Microscopy and Electron Holography of Magnetization Process in Ferrite Magnets
}

\author{
Toshiharu Aiso ${ }^{1, *}$, Daisuke Shindo ${ }^{1}$ and Takafumi Sato ${ }^{2}$ \\ ${ }^{1}$ Institute of Multidisciplinary Research for Advanced Materials, Tohoku University, Sendai 980-8577, Japan \\ ${ }^{2}$ NEC TOKIN Techno Service Ltd., Sendai 982-8510, Japan
}

\begin{abstract}
The magnetic domain structures and magnetization processes of anisotropic $\mathrm{Ba}$ and $\mathrm{Sr}$ ferrite magnets are investigated by Lorentz microscopy and electron holography. By utilizing a sharp magnetic needle made of a sintered Nd-Fe-B magnet in a transmission electron microscope, the magnetization process in ferrite magnets is visualized for the first time by in situ Lorentz microscopy. In the anisotropic Ba ferrite specimen, magnetization reversal occurs suddenly along the grain boundaries over a large volume, which includes the volumes of some grains located around the specimen edges. On the other hand, in the anisotropic Sr ferrite specimen, magnetization reversal is not observed due to the large coercivity of the specimen. During the magnetization process from the demagnetized state in the Sr ferrite specimen, the domain wall inside a grain moves gradually along the grain boundary and then stops at the grain boundary. [doi:10.2320/matertrans.MD200709]
\end{abstract}

(Received March 28, 2007; Accepted May 16, 2007; Published July 11, 2007)

Keywords: ferrite magnet, magnetization reversal, magnetization process, Lorentz microscopy, electron holography

\section{Introduction}

Hard ferrites having magnetoplumbite structures are major permanent magnetic materials because of their optimal performance-to-cost ratio and high chemical stability. ${ }^{1)}$ They have been widely used in various applications such as motors, speakers, toys, etc. In order to decrease the weights of motors used in automobiles and to improve the efficiencies of motors used in electric appliances, many efforts have been made to improve the magnetic properties of ferrite magnets in recent years. ${ }^{2-5)}$ In order to further improve the magnetic properties of ferrite magnets, such as coercivity, remanent flux density, and maximum energy product, it is essential to understand the magnetization process. Previously, we successfully employed electron holography to statically observe the changes in the magnetic domain structures in Ba ferrite magnets by using a specially designed electromagnet capable of applying external magnetic fields. ${ }^{6,7)}$ However, in order to understand the details of the magnetization process, it is necessary to observe the magnetization process dynamically. In this study, by utilizing a sharp magnetic needle made of a sintered Nd-Fe-B magnet for inducing a strong magnetic field within a transmission electron microscope (TEM), ${ }^{8,9}$ the magnetization processes in anisotropic $\mathrm{Ba}$ and $\mathrm{Sr}$ ferrite magnets were visualized by in situ Lorentz microscopy in combination with electron holography.

\section{Experimental Procedure}

Anisotropic $\mathrm{Ba}$ and $\mathrm{Sr}$ ferrite magnets provided by NEC Tokin Corp. were prepared by conventional powder metallurgy. ${ }^{1)}$ The mean grain sizes of the $\mathrm{Ba}$ and $\mathrm{Sr}$ ferrite magnets are approximately $1.4 \mu \mathrm{m}$. For both the ferrite magnets, the values of remanence $\left(B_{r}\right)$, coercivity $\left(H_{c}\right)$, and maximum energy product $\left[(B H)_{\max }\right]$ are listed in Table 1 . The samples were etched and polished by a focused ion beam technique (JEM-9310FIB); then, the thin foils were placed on carbon-

*Graduate Student, Tohoku University
Table 1 Magnetic properties of anisotropic $\mathrm{Ba}$ and $\mathrm{Sr}$ ferrite magnets.

\begin{tabular}{cccc}
\hline Specimen & $\begin{array}{c}B_{r} \\
(\mathrm{~T})\end{array}$ & $\begin{array}{c}H_{c} \\
(\mathrm{kA} / \mathrm{m})\end{array}$ & $\begin{array}{c}(B H)_{\max } \\
\left(\mathrm{kJ} / \mathrm{m}^{3}\right)\end{array}$ \\
\hline Ba ferrite & 0.41 & 179 & 30.4 \\
Sr ferrite & 0.42 & 251 & 33.6 \\
\hline
\end{tabular}

coated microgrids for TEM observations using a glass manipulator. ${ }^{10)}$ The reason for using this method, which is called the pickup method, is that the magnetic domain structures in the thin foil specimens prepared by the conventional focused ion beam method are considerably affected by the stray field that originates from the surrounding bulk portion. The pickup method enabled an accurate analysis of the magnetic domain structures in the thin foil specimens by eliminating the bulk portion. Taking into account the width of the interference fringe in the electron hologram, i.e., approximately $4 \mu \mathrm{m}$, the sizes of the thin foil specimens were maintained at approximately $4 \mu \mathrm{m} \times 4 \mu \mathrm{m} \times 100 \mathrm{~nm}$. Thus, the magnetic domain structures in the entire region could be observed. Both Lorentz microscopy and electron holography experiments were carried out using a JEM-3000F TEM with a field emission gun and a biprism. In the microscope, a special polepiece is designed for observing the magnetic materials; ${ }^{11)}$ consequently, the magnetic field of the objective lens at the specimen position can be reduced to $32 \mathrm{~A} / \mathrm{m}$. In order to carry out in situ observations, the specimen and a sharp magnetic needle made of a sintered $\mathrm{Nd}-\mathrm{Fe}-\mathrm{B}$ magnet were installed together in a special specimen holder controlled by a stepping motor and piezo drives, as schematically shown in Fig. $1 .^{8)}$ As the magnetic needle was brought closer to the specimen, the magnetization process was observed by Lorentz microscopy in the Fresnel mode; ${ }^{9)}$ for enabling clear observation, a Gatan 676 TV camera system was used. The remanence, coercivity, and maximum energy product of the magnetic needle used in this study were $1.39 \mathrm{~T}, 1.1 \mathrm{MA} / \mathrm{m}$, and $368 \mathrm{~kJ} / \mathrm{m}^{3}$, respectively. The magnetic field at the top of the needle was estimated by simulation to be approximately 


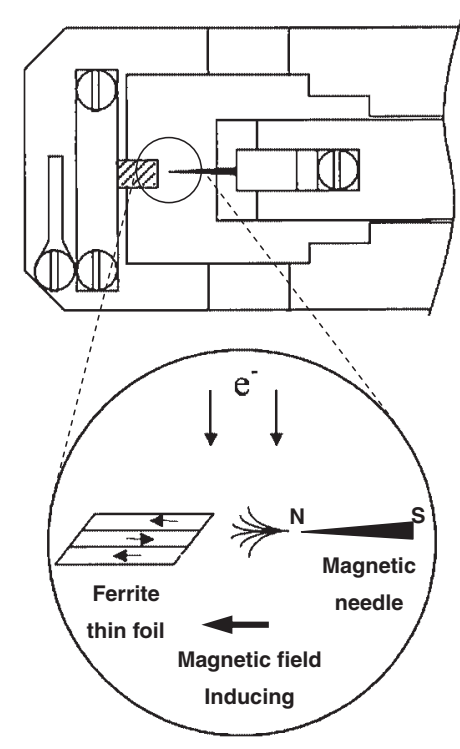

Fig. 1 Schematic illustration of a part of the specimen holder equipped with the sharp magnetic needle.
$560 \mathrm{kA} / \mathrm{m}$; the magnetization direction of the needle is shown in Fig. 1.9)

\section{Results and Discussion}

First, magnetization reversal was observed dynamically in the anisotropic Ba ferrite specimen, the coercivity of which is smaller than that of the anisotropic $\mathrm{Sr}$ ferrite specimen. Figure 2 shows Lorentz microscope images (left panels) and reconstructed phase images (right panels) of the anisotropic $\mathrm{Ba}$ ferrite specimen observed in the remanent state after applying an external magnetic field of $2 \mathrm{MA} / \mathrm{m}$ using an electromagnet [Figs. 2(a) and (b)] and after inducing a magnetic field along the opposite direction using the magnetic needle [Figs. 2(c) and (d)]. The black zonal area observed in the lower part of the specimen corresponds to the amorphous layer formed during the focused ion beam milling process. In the reconstructed phase images [Figs. 2(b) and (d)], the white lines indicate the specimen edges and grain boundaries; the small black and white arrows, the directions of the lines of magnetic flux in each grain. In Fig. 2(a), no
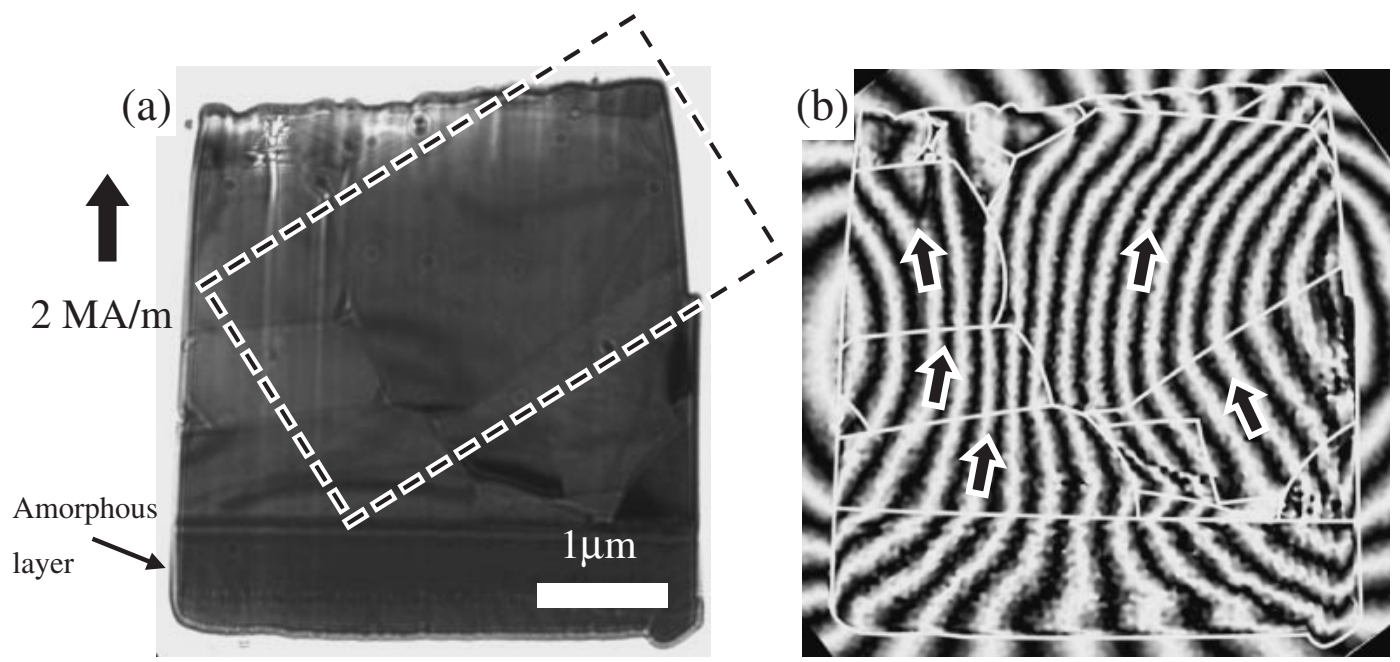

Direction of approach of a needle

(c)

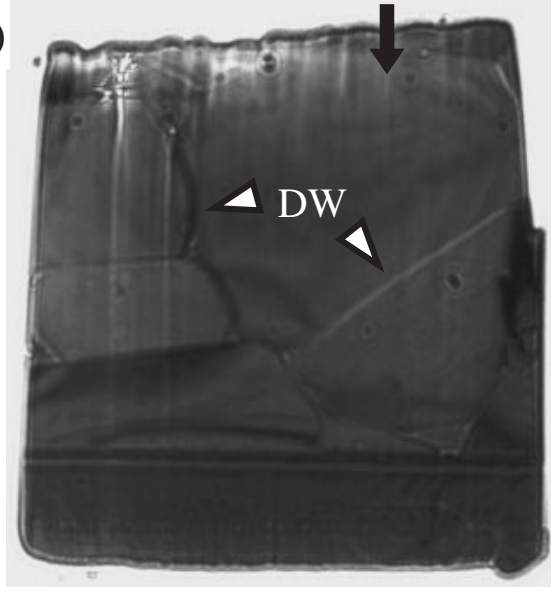

(d)

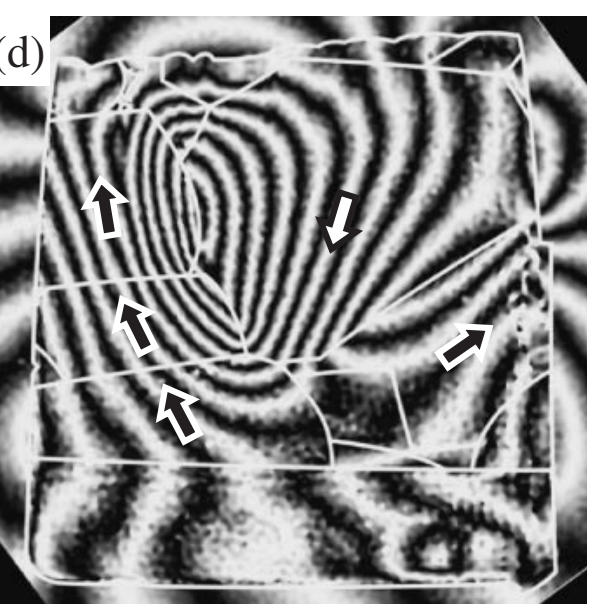

Fig. 2 Lorentz microscope images (left panels) and reconstructed phase images (right panels) of the anisotropic Ba ferrite specimen observed in the remanent state after (a), (b) applying an external magnetic field of $2 \mathrm{MA} / \mathrm{m}$ using an electromagnet and (c), (d) inducing a magnetic field along the opposite direction using the magnetic needle. In (c), DW indicates the domain wall (white arrowhead). The large black arrow in (a) and the small black arrow in (c) indicate the direction of the applied external magnetic field and the direction of approach of the magnetic needle, respectively. 

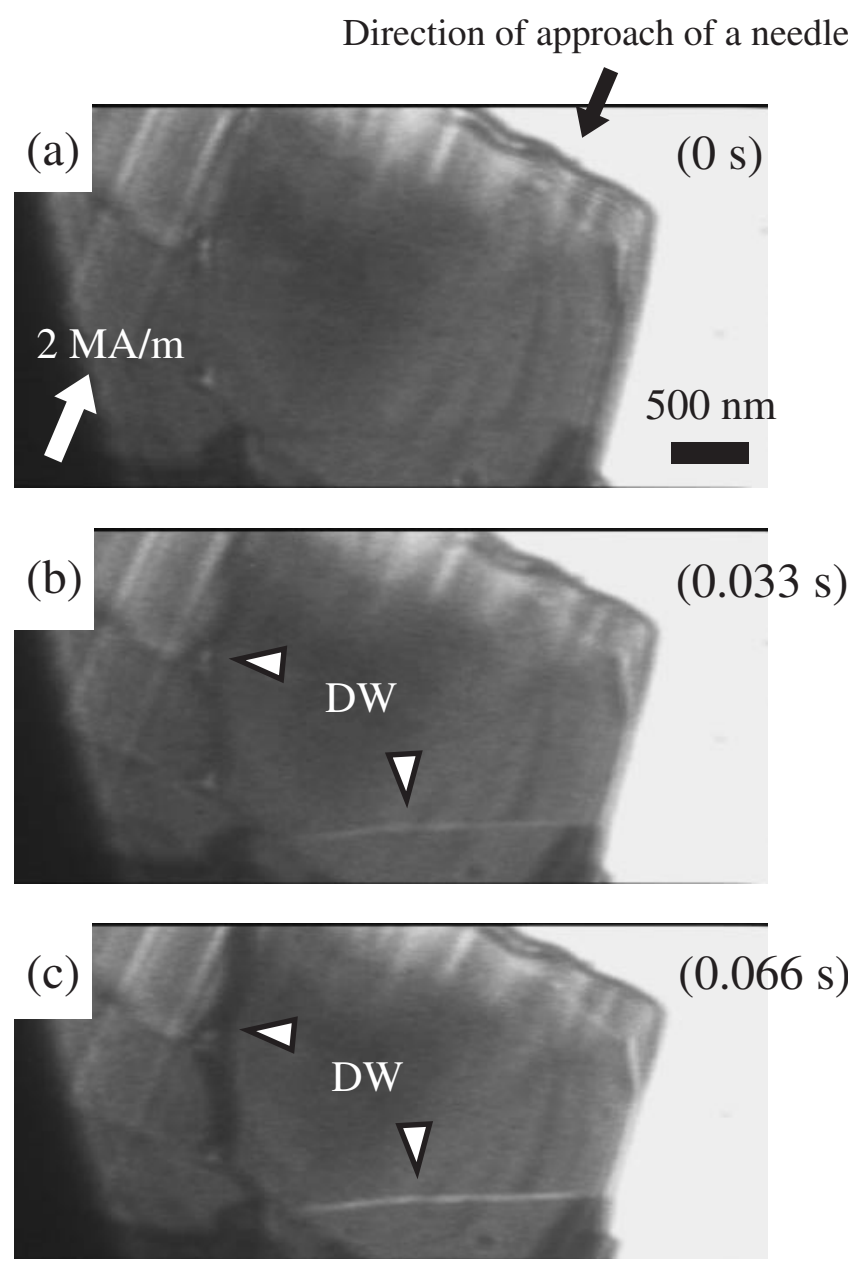

Fig. 3 Lorentz microscope images showing the magnetization reversal from the remanent state. The white and black arrows in (a) indicate the direction of the applied external magnetic field and the direction of approach of the magnetic needle, respectively. DW indicates the domain wall (white arrowhead).

white lines and black bands, which correspond to the domain walls, were observed at this field of view because the specimen was magnetized. When the magnetic needle was brought close to the specimen and then withdrawn, the domain walls indicated by the white arrowhead were observed at the grain boundary [Fig. 2(c)], and a reversal in the magnetization direction was observed in some grains including a large grain located around the specimen edges [Fig. 2(d)]. In order to understand this magnetization reversal in detail, the dynamic observation was carried out by in situ Lorentz microscopy in the area surrounded by the dashed lines shown in Fig. 2(a), as described below.

Figure 3 shows Lorentz microscope images indicating the magnetization reversal from the remanent state shown in Figs. 2(a) and (b); these images were captured from videotape. The passage of time is indicated in the top right corner. The speed of the needle was estimated to be approximately $25 \mathrm{~nm}$ per frame or $0.033 \mathrm{~s}$. By using the electromagnet, a magnetic field of $2 \mathrm{MA} / \mathrm{m}$ was applied to the specimen along a direction parallel to that indicated by the white arrow in frame (a) and then removed. On the other hand, the magnetic needle approaches the specimen along the direction indicated by the black arrow. By means of simulation, the magnetic field at the specimen position in frame (a) is approximately evaluated to be $120 \mathrm{kA} / \mathrm{m}$. Since the specimen was magnetized, no domain walls were observed until frame (a). However, the domain walls suddenly appeared along the grain boundaries immediately after frame (a), as shown in frames (b) and (c). The domain walls were faintly observed in frame (b) and clearly observed in frame (c). Although the magnetic needle was brought closer to the specimen, on which a magnetic field of approximately $160 \mathrm{kA} / \mathrm{m}$ could be applied as estimated by simulation, and then withdrawn, the domain wall did not move. It should be noted that magnetization reversal occurs over a relatively large volume, which includes the volumes of some grains located around the specimen edges. A reversed domain is formed around the specimen edges due to enhanced stray fields at those locations. It is considered that a large reversed domain can be formed more easily than a small one by minimizing the magnetic energy. Although dynamic observation in the anisotropic Sr ferrite specimen was attempted in a similar manner, magnetization reversal was not observed, probably due to the large coercivity of the specimen.

Second, the magnetization process from the demagnetized state was observed dynamically in the anisotropic Sr ferrite specimen. Figure 4 shows Lorentz microscope images (left panels) and reconstructed phase images (right panels) of the anisotropic Sr ferrite specimen observed in the demagnetized state [Figs. 4(a) and (b)] and the remanent state after inducing a magnetic field using the magnetic needle [Figs. 4(c) and (d)]. The direction of approach of the magnetic needle is indicated by the large black arrow in Fig. 4(c). The zonal area observed in the upper part of the specimen corresponds to the carbon layer that was deposited and formed during the focused ion beam milling process. In Fig. 4(a), the domain wall indicated by the white arrowhead was observed inside a large grain, the size of which is much larger than the critical size for single domain particles, i.e., approximately $1 \mu \mathrm{m} .{ }^{12}$ ) The magnetic domains in the large grain were divided by a $180^{\circ}$ wall, as indicated by the dotted line in Fig. 4(b). When the magnetic needle was brought closer to the specimen and then withdrawn, the domain wall moved to the grain boundary [Fig. 4(c)], and the multidomain structure inside the large grain disappeared [Fig. 4(d)]. In order to understand this magnetization process in detail, the motion of the domain wall was observed by in situ Lorentz microscopy in the area surrounded by the dashed lines shown in Fig. 4(a), as described below.

Figures 5(b)-(i) show Lorentz microscope images of the magnetization process from the demagnetized state. The direction of approach of the magnetic needle is indicated by the black arrow in Fig. 5(c). The distance between the magnetic needle and the upper edge of the specimen is indicated at the upper right part of each figure. In Fig. 5(a), the grain boundaries are schematically illustrated. In the demagnetized state shown in Fig. 5(b), the lower end of the domain wall touched the grain boundary, while the upper end touched the carbon layer. As the needle was brought closer, the domain wall moved slightly to the left, and the lower end of the domain wall was located at the junction of the grain boundary [Fig. 5(c)]. In Fig. 5(d), the upper part of the domain wall moved to the left, leading to an increase in the 

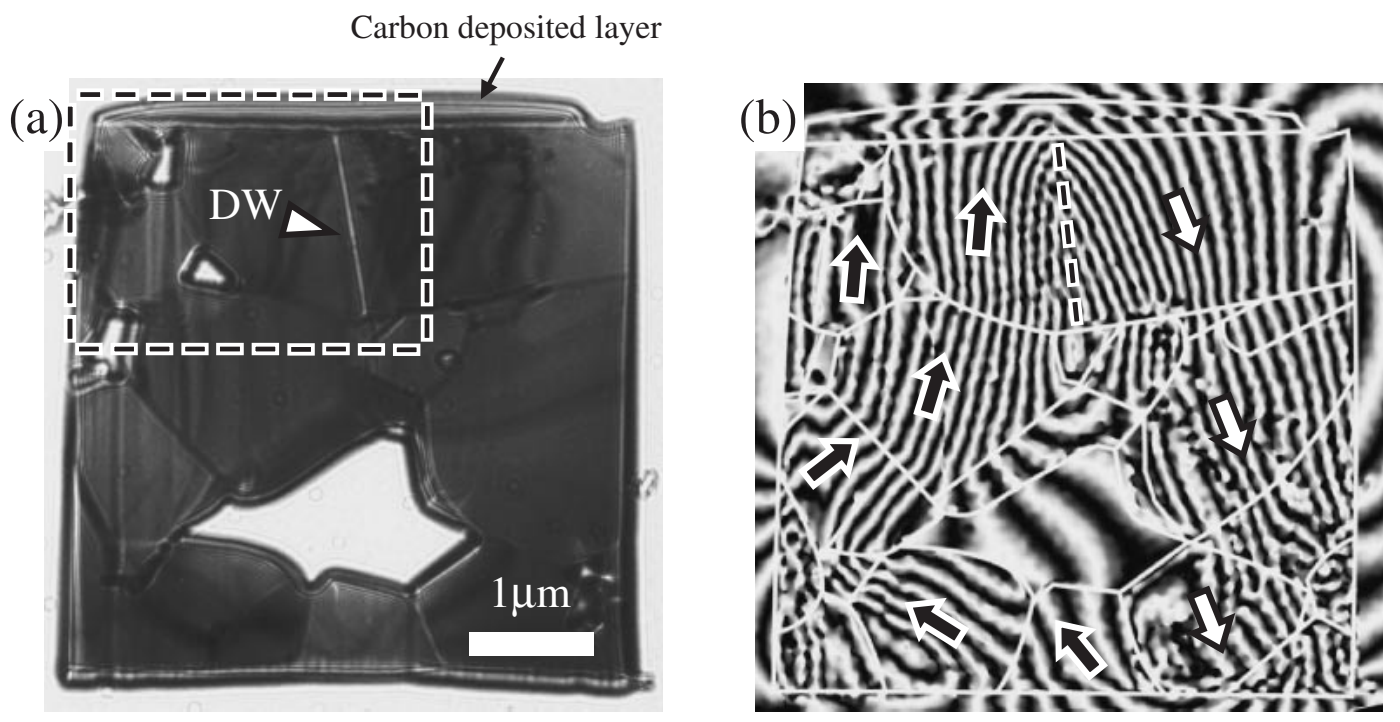

Direction of approach of a needle
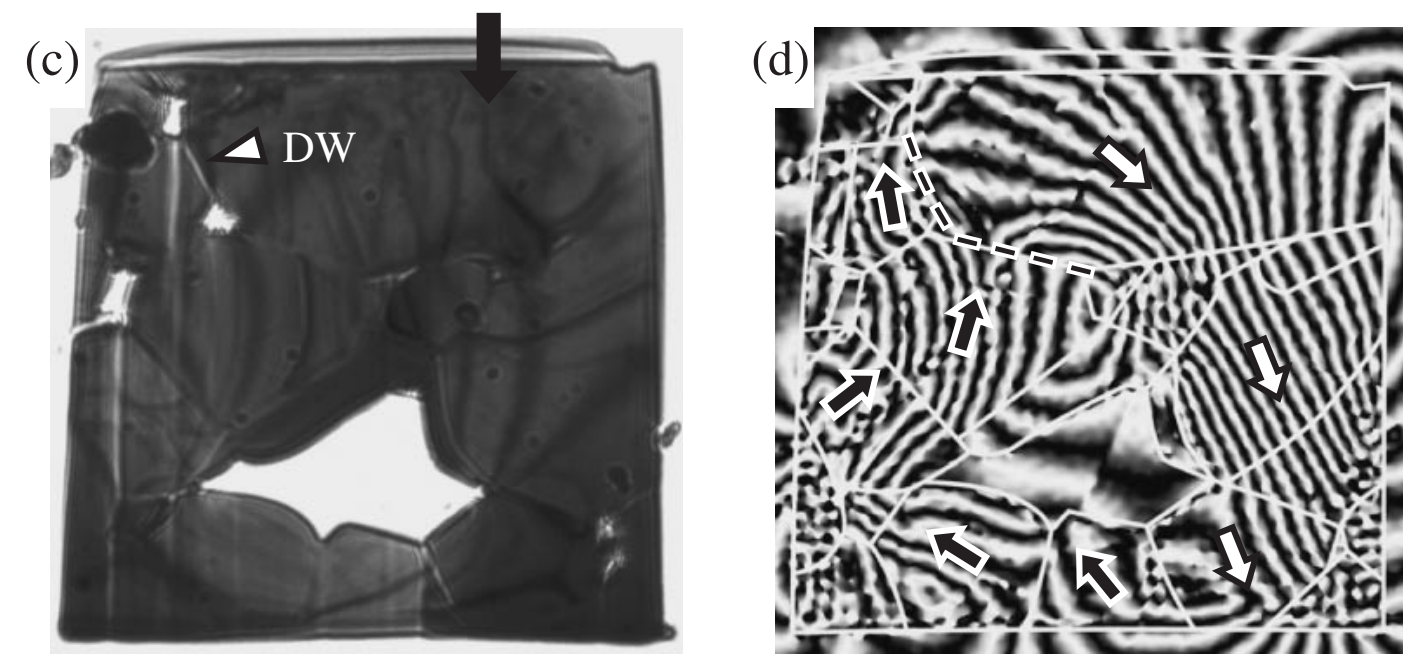

Fig. 4 Lorentz microscope images (left panels) and reconstructed phase images (right panels) of the anisotropic Sr ferrite specimen observed in the (a), (b) demagnetized state and (c), (d) remanent state after inducing a magnetic field using the magnetic needle. In (a) and (c), DW indicates the domain wall. The small white and black arrows in (b) and (d) indicate the directions of the lines of magnetic flux in each grain.

area of the domain magnetized parallel to the applied magnetic field, while the lower part did not move at the junction of the grain boundary due to domain wall pinning. It is noted that the domain wall is slightly curved. It can be considered that the applied magnetic field exerts pressure perpendicular to the domain wall. With the increase in the magnetic field, the lower part of the domain wall was dragged along the grain boundary and glided gradually to new stable positions [Figs. 5(e)-(h)]. Finally, the domain wall stopped at the grain boundary and the pores, as shown in Fig. 5(i). Although the magnetic needle was brought closer to the specimen, on which a magnetic field of approximately $160 \mathrm{kA} / \mathrm{m}$ could be applied as estimated by simulation, and was then withdrawn, the domain wall did not move. As described above, in contrast to the magnetization reversal from the remanent state, domain wall motion was observed continuously during the magnetization process from the demagnetized state. However, the domain wall was eventually located at the grain boundaries due to strong domain wall pinning.

\section{Conclusions}

The magnetization process in the ferrite magnets was investigated by in situ observations using Lorentz microscopy and electron holography. The results are summarized as follows:

(1) In the remanent state of the anisotropic $\mathrm{Ba}$ ferrite specimen after the specimen is magnetized by the electromagnet, no domain walls are observed. With an increase in the magnetic field, magnetization reversal occurs suddenly along the grain boundaries for a large volume, which includes the volumes of some grains located around the specimen edges, due to the presence of stray fields and minimization of the magnetic energy. Magnetization reversal is not observed in the anisotropic $\mathrm{Sr}$ ferrite specimen. This is considered to result from the difference of their coercivity.

(2) In the demagnetized state of the anisotropic $\mathrm{Sr}$ ferrite specimen, the domain wall is observed inside a large grain, the size of which is much larger than the critical size for single domain particles. With an increase in the magnetic 

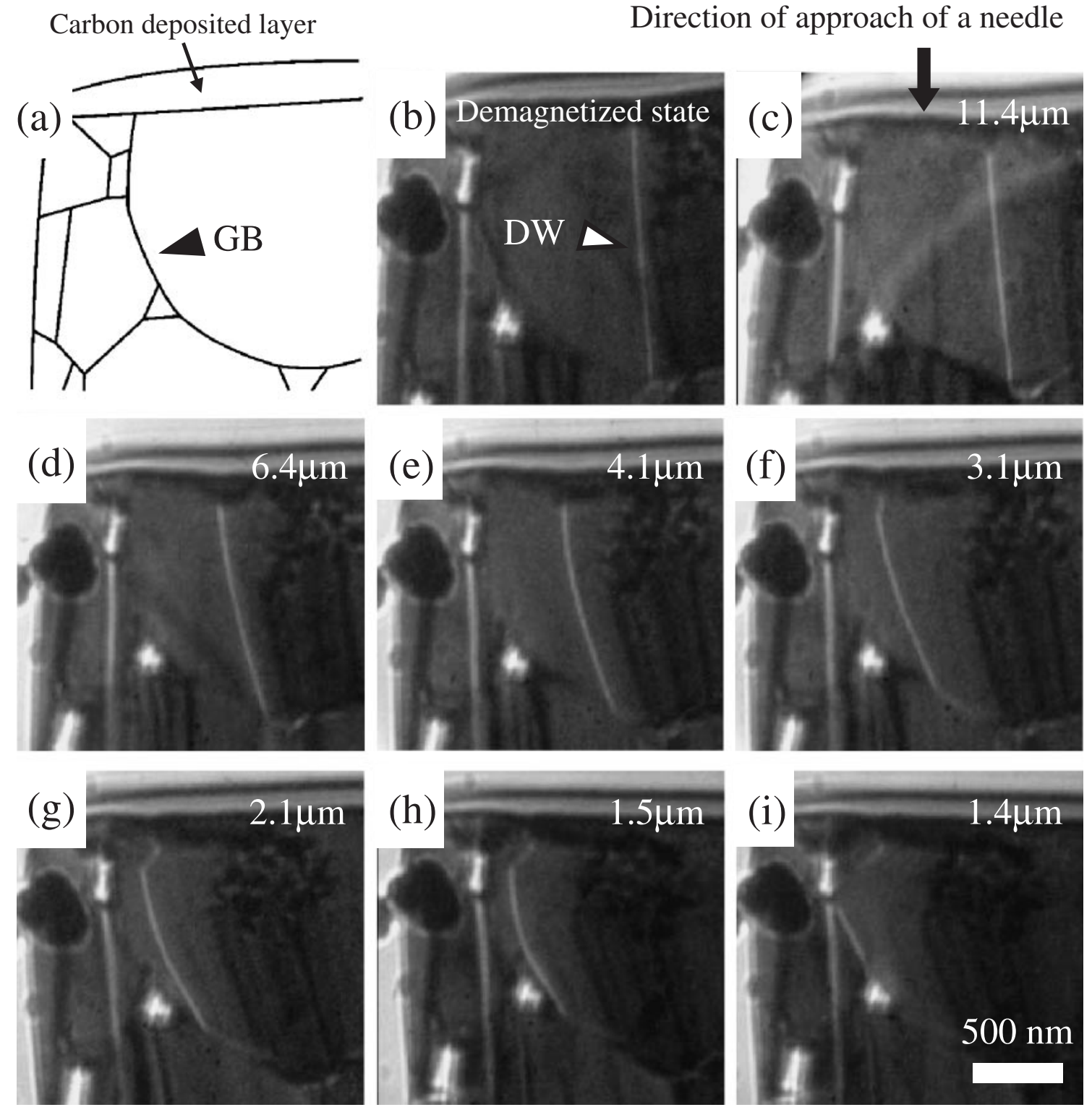

Fig. 5 (a) Schematic illustration showing the grain boundary of the anisotropic Sr ferrite specimen. GB indicates the grain boundary (black arrowhead). (b)-(i) Lorentz microscope images directly showing domain wall motion during the magnetization process from the demagnetized state in the anisotropic Sr ferrite specimen. In (b), DW indicates the domain wall (white arrowhead).

field, the domain wall moves gradually along the grain boundary and stops at the grain boundary. The domain wall cannot move across the grain boundary due to strong domain wall pinning at the grain boundary.

\section{Acknowledgments}

This work was partly supported by a Grant-in-Aid for Scientific Research (A) from the Japan Society for the Promotion of Science.

\section{REFERENCES}

1) H. Kojima: Ferromagnetic Materials, vol. 3 (North-Holland Publishing Company, 1982) 305.

2) H. Taguchi, F. Hirata, T. Takeishi and T. Mori: Proc. 6th Int. Conf. on Ferrites, Tokyo, 1992, p. 1118.
3) H. Taguchi, Y. Minachi, K. Masuzawa and H. Nishio: Proc. 8th Int. Conf. on Ferrites, Kyoto, 2000, p. 405.

4) Y. Kubota, T. Takami and Y. Ogata: Proc. 8th Int. Conf. on Ferrites, Kyoto, 2000, p. 410.

5) F. Kools, A. Morel, R. Grössinger, J. M. Le Breton and P. Tenaud: J. Magn. Magn. Mater. 242-245 (2002) 1270.

6) T. Aiso, D. Shindo and T. Sato: Collected Abstracts of the 2006 Spring Meeting of the Japan Inst. Metals (2006) p. 379.

7) T. Aiso, D. Shindo and T. Sato: Collected Abstracts of the 2006 Autumn Meeting of the Japan Inst. Metals (2006) p. 465.

8) D. Shindo, Y.-G. Park, Y. Gao and H. S. Park: J. Appl. Phys. 95 (2004) 6521-6526.

9) H. S. Park, Y.-G. Park, Y. Gao, D. Shindo and M. Inoue: J. Appl. Phys. 97 (2005) 033908-1-033908-4.

10) T. Suzuki, N. Endo, E. Okunishi and T. Kuba: JEOL News 38 (2003) 30.

11) D. Shindo, Y.-G. Park, Y. Murakami, Y. Gao, H. Kanekiyo and S. Hirosawa: Scr. Mater. 48 (2003) 851-856.

12) C. Kittel: Phys. Rev. 70 (1946) 965. 